\title{
Eye Movements Matter, But Why? Psychophysiological Correlates of EMDR Therapy to Treat Trauma in Timor-Leste
}

\author{
Sarah J. Schubert \\ Christopher W. Lee \\ Peter D. Drummond \\ Murdoch University, Australia
}

\begin{abstract}
This preliminary study examined the physiological correlates of eye movement desensitization and reprocessing (EMDR) therapy when effectively used to treat trauma symptoms in a postconflict, developing nation, Timor-Leste. Participants were 20 Timorese adults with posttraumatic stress disorder (PTSD) symptoms treated with EMDR therapy. PTSD, depression, and anxiety decreased significantly after an average of $4.15(S D=2.06)$ sessions. Continuous measures of heart rate, skin conductance, and respiration were collected during the first and last desensitization sessions. Physiological activity decreased in EMDR desensitization sessions, and eye movement sets were associated with an immediate significant decrease in heart rate and an increase in skin conductance, consistent with an orienting response. This response habituated within and across eye movement sets. These findings suggest that effective EMDR therapy is associated with de-arousal within sessions and that eye movement sets are associated with distinct physiological changes that may aid memory processing. The findings offer insight into the working mechanisms of EMDR when used to treat PTSD symptoms in a real-world, cross-cultural, postwar/ conflict setting.
\end{abstract}

Keywords: EMDR therapy; mechanisms of action; psychophysiology; orienting response; REM hypothesis; Timor-Leste.

$\mathbf{E}$ ye movement desensitization and reprocessing therapy (EMDR) and trauma-focused cognitive behavioral therapy (CBT) are recognized internationally as the two most efficacious treatments for posttraumatic stress disorder (PTSD; Australian Centre for Posttraumatic Mental Health, 2013; World Health Organization, 2013). Still, for both therapies, evidence for their effectiveness in non-Western, postwar/conflict, real-world settings is scarce, and the exact mechanisms of change underlying effective treatment remain unknown (Schnyder et al., 2015). What is known is that dysregulated psychophysiological arousal underlies symptoms of PTSD: persistent hyperarousal (i.e., increased resting heart rate), exaggerated responses to startling sounds, and elevated responses to external and internal trauma reminders (Pole, 2007). The increased arousal has been found to disturb sleep and memory processing (Stickgold, 2002). Effective PTSD treatment with both therapies is associated with physiological de-arousal from pre- to posttreatment, reduced psychophysiological activity when trauma memories are recalled (Sack, Hofmann, Wizelman, \& Lempa, 2008), and improvements in sleep quality (Raboni, Tufik, \& Suchecki, 2006). However, the therapeutic processes that lead to physiological changes and PTSD symptom reduction are not entirely understood.

EMDR therapy (Shapiro, 2001) emphasizes the role of memory and the information processing system in treatment of symptoms resulting from unresolved life experiences. EMDR consists of eight phases, structured in a way to process past, present, and future ramifications of unresolved or dysfunctionally stored memories. EMDR is an integrative treatment with many underlying processes: cognitive restructuring, mindfulness, free association, psychological distancing, perceived mastery, somatic awareness, and conditioning (Oren $\&$ Solomon, 2012). A distinguishing difference between EMDR and exposure therapy is that eye movements (EMs) are used as a 
dual-attention task during desensitization of trauma memories. Early reviews (Cahill, Carrigan, \& Frueh, 1999) and meta-analyses (Davidson \& Parker, 2001) questioned beneficial effects of the EM element. However, a recent meta-analysis has demonstrated that the EMs significantly add to the beneficial treatment effects of EMDR (Lee \& Cuijpers, 2013).

EM may evoke orienting responses and/or REMlike mechanisms. The orienting response theory of EMDR states that EMs, or other dual-attention stimuli, elicit an orienting response with associated physiological de-arousal that enhances processing of trauma material (Söndergaard \& Elofsson, 2008). The orienting response is an autonomic response evoked by any new stimulus that is assessed for threat and reflects an overall increase in physical arousal. With repeated presentation of the same nonthreatening stimulus, the orienting response habituates quickly (within 10 seconds), indicating a reduction in physiological response to that stimulus (Bradley, 2009).

REM hypotheses of EMDR posit that the EMs, possibly through repeated orienting responses, shift the brain into a memory processing mode similar to REM sleep, which aids integration of episodic traumatic memories into general semantic networks (Stickgold, 2002). REM sleep plays an important role in internal information processing and memory consolidation. The orienting response and REM sleep both have recognizable physiological characteristics. For example, distinct indices of an orienting response include a biphasic initial startle response and cholinergic activation indexed by skin conductance responses and decrease in heart rate followed by a stress or relaxation response depending on the presence of threat (Bradley, 2009; Sokolov \& Cacioppo, 1997). Although the autonomic profile of REM sleep is more complex than an orienting response, features of REM sleep are momentary cholinergic activation; startle responses; rapid, shallow breathing; and temperature increases in the extremities (Stickgold, 2002). Interestingly, both orienting responses and REM sleep involve EMs (Wright \& Ward, 2008). Acknowledging that the type of EMs in orienting responses (visual or smooth pursuit) and REM sleep (saccadic) may differ, a way of examining orienting response and REM hypotheses of EMDR therapy is to study changes in physiological activity during EMDR desensitization sessions.

Few studies have investigated psychophysiological concomitants during EMDR. Two uncontrolled studies that measured physiological changes throughout EMDR therapy of patients with PTSD found a crosssession trends of psychophysiological de-arousal, and EMs were associated with increased parasympathetic nervous system activity, that is, decreased heart rate and increased heart rate variability, decreased sympathetic nervous system activity (reflected by decreased skin conductance and increased finger temperature), but also increased respiration rate (Elofsson, von Schéele, Theorell, \& Söndergaard, 2008; Sack, Lempa, Steinmetz, Lamprecht, \& Hoffman, 2008). Authors highlighted that some physiological data associated with EMs fit with orienting response theory (i.e., reduced heart rate immediately as EMs began), yet others contradicted (i.e., increased respiration rate and skin temperature). However, these contradictory physiological responses were characteristic of REM sleep. Thus, the EMs in EMDR may create orienting responses and a REM-like state. However, to attribute any changes to EMDR therapy, or the EMs, a comparison control is needed. Previously, Wilson, Silver, Covi, and Foster (1996) recorded physiological changes during EMDR treatment of traumatic memories compared to two other treatments. They reported that heart rate and blood pressure decreased and finger temperature increased during EMDR but not in control groups. During EMs, heart rate decreased, respiration changes were described as matching the EM rhythm and became shallow, and the galvanic skin response increased in the first $5-10$ seconds then decreased within the EM set. In a nonclinical sample where negative autobiographical memories were treated with EMDRwith-EMs or with a similar procedure but without EMs (Schubert, Lee, \& Drummond, 2011), a similar physiological profile was seen: de-arousal across the treatment session; EMs were associated with immediate significant reductions in heart rate; heart rate variability and respiration rate increased across the set; and, although skin conductance decreased across the EMs set, short bursts in skin conductance occurred as EMs began. Skin conductance response number and size were more frequent in the EMDR-with-EM than the EMDR-without-EM condition, and responses habituated within EM sets and across the session, characteristic of an orienting response.

The limited research that has measured physiological activity during EMDR therapy has been conducted in Western treatment settings. Whether the psychophysiological correlates of EMDR therapy might apply cross-culturally, and/or in treatment of PTSD postwar/ conflict in a real-world setting, has yet to be investigated. Thus, the aim of this study was to examine the psychophysiological correlates of EMDR therapy when used to treat symptoms consistent with PTSD in a real-world, postwar/conflict setting in Timor-Leste. Timor-Leste is a half-island country north of Australia that was invaded and occupied by 
Indonesian military (1975-1999). This time was characterized by allegations of extensive human rights violations, including mass killings, torture, systematic rape, and arbitrary detention. In 1999, a vote for independence was followed by widespread destruction of infrastructure by pro-Indonesian militias and $80 \%$ of the East Timorese population was displaced (Modvig et al., 2000). We recently reported that EMDR therapy was successful in treating PTSD symptoms in adults in Timor-Leste (Schubert et al., 2016). In this study, it was hypothesized that physiological de-arousal would occur within EMDR treatment sessions in Timor-Leste and that psychophysiological responses associated with the EMs would be similar to those seen in Western settings: decreased heart rate as EMs began, decreased skin conductance within EM sets, respiration rate increases in EM sets, along with skin conductance responses that habituate in size and number in EM sets characteristic of an orienting response.

\section{Method}

This study was designed to examine the psychophysiological correlates of EMDR therapy to treat PTSD symptoms in Timor-Leste. The study was designed so that following an initial 2-hour assessment, which included teaching stabilization techniques, participants served as their own 2-week waitlist control. Subsequently, they engaged in EMDR therapy, with posttreatment assessments 2 weeks after the final treatment session, and follow-up at 3 months (Schubert et al., 2016). During the first and last EMDR treatment sessions, where desensitization of traumatic memories took place, physiological activity was recorded. In the assessment, participants gave written consent. The Murdoch University Human Research Ethics Committee, Australia, and the Cabinet of Health Research and Development under the Ministry of Health in Timor-Leste approved the research.

\section{Participants and Procedure}

Participants were from an EMDR treatment trial (Schubert et al., 2016). In the trial, 28 participants were referred primarily through Timorese community development and health nongovernmental organizations, Australian People for Health, Education and Development Abroad, and Psychosocial Recovery and Development in East Timor. Inclusion criteria were Timorese, aged 18-65 years, had experienced traumatic events at least 3 months before study entry, reported symptoms consistent with PTSD with a Trauma Symptom Scale score of $\geq 2$ using the Harvard Trauma Questionnaire (HTQ; Mollica et al., 1992). Exclusion criteria were history of eye disease or blindness. Included were 23 participants ( 16 female, $69.6 \%$ ) with a mean age of $30.52(S D=11.29)$ years. Excluded participants were four who scored $<2$ on the HTQ Trauma Symptom Scale, and one whose memories of traumatic experiences were not associated with hyperarousal, and symptom presentation was more consistent with depression and anxiety than PTSD. Prior to commencing EMDR treatment, two participants dropped out from the wait period and one dropped out after commencing therapy (see Figure 1 for flow diagram of participants and attrition for this treatment trial).

Thus, for 20 participants, EMDR therapy followed Shapiro's (2001) protocol and included all eight treatment phases: (1) client history and assessment, (2) preparation, (3) target assessment, (4) desensitization, (5) installation of positive cognitions, (6) body scan, (7) closure, and (8) reevaluation. In this study, Phases 1-2 occurred in the initial assessment session. Target memories were also identified throughout the assessment. Following an explanation of the research rationale and EMDR procedure, participants were asked which traumatic experience they would like to or felt most comfortable processing first in treatment. Identification of possible initial target memories occurred in the assessment session to prepare for the first treatment session. This treatment session began with Phase 3, target assessment, and also included desensitization (Phase 4). If time allowed, the first treatment session also included Phases 5-7. EMDR treatment sessions were 1-1.5 hours long. Individual needs of participants determined the length of treatment.

\section{Therapists and Fidelity}

Two therapists provided EMDR therapy. Both were clinical psychologists and accredited EMDR Association of Australia consultants. The translator was a local Timorese person with tertiary education and experience in community development and health but no formal psychology training. Therapists and the translator followed a step-by-step EMDR protocol. When consent was provided, treatment sessions were video recorded. An EMDR International Associationapproved consultant randomly selected 12 sessions and rated them on a 27-item EMDR fidelity checklist. Items were scored on a 7 -point scale $(1=$ poor; $7=$ excellent), and the average rating for each session was $6.08(S D=0.67)$.

\section{Psychophysiological Assessment}

Changes in heart rate, skin conductance, and respiration rate were recorded continuously throughout the 


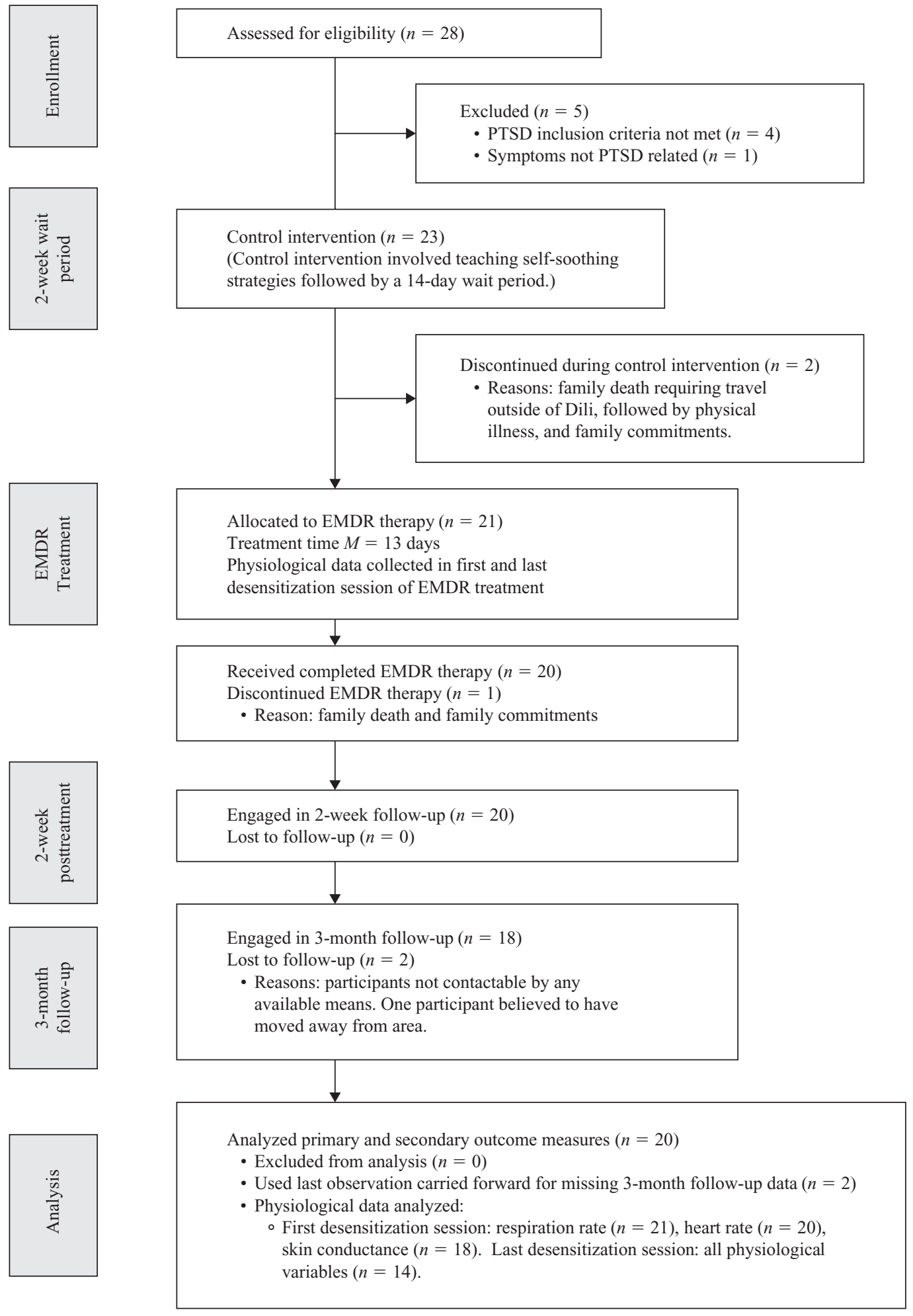

FIGURE 1. Flow diagram for EMDR therapy trial in Timor-Leste. PTSD = posttraumatic stress disorder.

first and last sessions where desensitization of traumatic memories took place. These variables were chosen because they could be measured noninvasively, without interference to treatment. At the beginning of the session, following placement of sensors and electrodes, a 5-minute adaptation period occurred to allow physiological activity to stabilize, then treatment began, and data was acquired during the entire session.

Electrocardiograms were recorded using an ECG100C Electrocardiogram Amplifier (BIOPAC Systems Inc.). A three-lead configuration was used with $\mathrm{Ag}-\mathrm{AgCl}$ snap electrodes (8-mm diameter) on 
the inner aspect of both forearms and the right ankle, secured with disposable $3 \mathrm{M}$ Red Dot foam tape and sticky gel Ag/ AgCl eyelet stud electrodes. Skin conductance was measured using a BIOPAC Galvanic Skin Response Amplifier GSR100C and was recorded by means of constant voltage $(0.5 \mathrm{~V}$, set to a gain of $2 \mu \mathrm{S}$ per volt) using $\mathrm{Ag} / \mathrm{AgCl}$ electrodes (8- $\mathrm{mm}$ internal diameter) filled with electrode gel. Electrodes were attached to the second phalanx of the ring and middle fingers on the nondominant hand. Respiration was measured using a BIOPAC General Purpose Transducer Amplifier DA100C with a differential pressure transducer (TSD160B). From this, $1.5 \mathrm{~mm}$ tubing was Luer-locked to sensor tubing attached to a BIOPAC RX110 self-inflating pressure pad transducer that was taped and strapped to the chest to record respiration. All sites were cleaned with an alcohol wipe to ensure good electrical contact between electrodes and skin. It was not possible to control ambient temperature or humidity. Signals were sampled 1,000 times per second via a BIOPAC MP100 data acquisition system and were averaged using AcqKnowledge software 3.9.0 (BIOPAC Systems Inc.). Processing of psychophysiological signals is outlined in previous research (Schubert et al., 2011, p. 4).

\section{Data Analysis}

To assess psychophysiological changes within sessions, the first 30-second period immediately prior to the desensitization phase was compared to the 30-second period following desensitization. Change was also examined by comparing physiological responses during the first three and last three EM sets of the session. To assess physiological changes during EM stimulation sets, the following measurement periods were defined: (a) 10-second interval prior to stimulation; (b) first 10 seconds of EM stimulation; (c) middle of stimulation, defined as the difference between the first and last10 seconds of each set; and (d) last $10 \mathrm{sec}$ onds of stimulation. The therapist, with a handheld event marker, marked all measurement periods and the start and end of EM sets in physiological files as data acquisition took place.

Some physiological data was missing in the first treatment session because there were difficulties with the power source in Timor-Leste. Because of electrical noise, skin conductance data was unusable for three participants and heart rate was also unusable for one participant. For all other participants, physiological equipment was powered through a deep cycle battery. Physiological data was available for 14 of the 20 participants who completed therapy in their last desensitization session, as treatment involved only one desensitization session for five participants, and physiological activity could not be recorded for one other participant.

Multivariate repeated measures analysis of variances (ANOVAs) that incorporated planned contrasts to examine a priori hypotheses were used to examine significant effects for within-session pre- and postphysiological changes and changes during EM stimulation periods in the last session in which desensitization took place. Specifically, hypotheses were significant decreases in physiological activity pre- and posttreatment session; significant decrease in heart rate from pre-EM stimulation to the first 10 seconds of EM sets, with this decrease remaining significantly lower than pre-EM across the EM set; significant decreases in skin conductance from pre-EM stimulation to the middle and end of EM sets; significant increase in respiration rate from the beginning to the end of EM sets; and significant increase in number and size of skin conductance responses in the first 10 seconds of EM sets compared to the middle and end of EM sets. Because of missing physiological data for some participants in their first desensitization session, univariate repeated measures ANOVAs which incorporated planned contrasts were used to examine significant effects in EM stimulation periods, and paired $t$ tests were used to investigate within session pre- and postphysiological changes. Huynh-Feldt corrected statistics were reported when the assumption of sphericity was violated. Partial eta squared $\left(\eta_{\mathrm{p}}^{2}\right)$ was used to report effect sizes for ANOVAs and Cohen's $d$ for planned contrasts. Being a confirmatory investigation, with analyses conducted on a priori hypotheses, one-tailed planned contrasts are reported. An overall significance level was an alpha level of .05. Data were analyzed using IBM SPSS statistics Version 21.

\section{Results}

The average number of treatment sessions was 4.14 $(S D=2.06$, range $=1-10$ sessions $)$ over an average of $12.95(S D=8.45)$ days. The mean number of traumatic events experienced reported by participants on the HTQ was 7.09 ( $S D=2.31$ ), and an average of 7.35 years (range 3 months to 24 years) had passed since targeted traumatic events occurred.

\section{Psychophysiological Trends Within EMDR Desensitization Sessions}

In the first treatment session, significant decreases were seen in the period from before to after 
TABLE 1. Means, Standard Deviations, and Changes in Physiological Variables During the 30-Second Rest Period Pre- and Postdesensitization in the First and Last Treatment Sessions

\begin{tabular}{|c|c|c|c|c|c|c|c|c|}
\hline \multirow{2}{*}{$\begin{array}{l}\text { Treatment } \\
\text { Session }\end{array}$} & \multirow[b]{2}{*}{ Variable } & \multirow[b]{2}{*}{$n$} & \multicolumn{2}{|c|}{ Presession } & \multicolumn{2}{|c|}{ Postsession } & \multirow{2}{*}{$\frac{\text { Statistical Comparison }}{t(d f)}$} & \multirow[b]{2}{*}{ Effect Size $^{\mathrm{a}}$} \\
\hline & & & M & $S D$ & M & $S D$ & & \\
\hline \multirow[t]{3}{*}{ First } & $\mathrm{HR}$ (bpm) & 20 & 84.32 & 13.45 & 81.46 & 11.62 & $2.31(19)$ & $0.23^{\star}$ \\
\hline & $S C(\mu S)$ & 18 & 0.24 & 0.13 & 0.20 & 0.11 & - & $n s^{\mathrm{b}}$ \\
\hline & RR (bpm) & 21 & 23.21 & 6.02 & 17.81 & 7.24 & $3.33(20)$ & $0.82^{\star \star}$ \\
\hline \multirow[t]{3}{*}{ Last } & $\mathrm{HR}$ (bpm) & 14 & 83.54 & 12.06 & 81.89 & 12.74 & - & ns \\
\hline & $S C(\mu S)$ & 14 & 0.31 & 0.11 & 0.25 & 0.10 & $3.90(13)$ & $0.62^{\star \star \star}$ \\
\hline & $\mathrm{RR}$ (bpm) & 14 & 21.96 & 5.35 & 20.32 & 4.89 & - & ns \\
\hline
\end{tabular}

Note. $\mathrm{HR}(\mathrm{bpm})=$ heart rate (beats per minute); $\mathrm{SC}=$ skin conductance; $\mu \mathrm{S}=$ microsiemens; $\mathrm{RR}(\mathrm{bpm})=$ respiratory rate (breaths per minute); $n s=$ nonsignificant.

${ }^{\mathrm{a} C o h e n ' s} d$.

${ }^{\mathrm{b}} \mathrm{SC}$ decrease approached significance, $t(17)=1.73, p=.051, d=.28$.

${ }^{\star} p<.05 .{ }^{\star \star} p<.01 .{ }^{\star \star \star} p<.001$.

desensitization of trauma memories for heart and respiration rate (Table 1). In addition, the decrease in skin conductance approached significance, $t(17)=$ $1.73, p=.051, d=.28$. For the last session in which trauma memories were desensitized, Pillai's trace indicated a significant overall effect of time on physiological variables, $\mathrm{V}=.61, F(3,11)=5.84, p=.012$, $\eta_{\mathrm{p}}^{2}=.61$. In this session, skin conductance decreased significantly. Changes in respiration and heart rate were not significant.

\section{Psychophysiological Changes During Eye Movement Stimulation}

For the last treatment session in which desensitization occurred, Pillai's trace indicated a significant effect of time on physiological variables for the first, $\mathrm{V}=.839, F(9,117)=5.05, p<.001, \eta_{\mathrm{p}}{ }^{2}=.280$, and last, $\mathrm{V}=.772, F(9,117)=4.51, p<.001, \eta_{\mathrm{p}}{ }^{2}=.257$, EM sets of the session. Significant univariate ANOVAs with planned contrasts revealed that heart rate decreased significantly within the first and last EM sets in the first desensitization session and within the last EM sets in the last treatment session (Table 2). The decreases in heart rate seen in the first three EM sets of the last treatment session approached significance, $F(1.46,19)=3.84, p=.051, d=228$. Overall, heart rate decreased significantly when EM sets began, and heart rate remained significantly lower than prestimulation during the middle and end of EM sets.

Skin conductance also decreased within EM sets, with significant decreases within the first EM sets in the first desensitization session, and in both the first and last EM sets in the last treatment session where desensitization of memories took place. Interestingly, skin conductance decreased within EM sets but, unlike heart rate, skin conductance did not decrease significantly within the first 10 seconds of EMs in any measurement period. Overall, respiration rate did not change significantly within EM sets.

The Number and Amplitude of Skin Conductance Responses Within Eye Movement Sets

The number of skin conductance responses per 10 seconds and the size of these responses were calculated for each measurement period (i.e., the start, middle, and end) in the first and last three EM sets of the first and last treatment sessions where desensitization of memories took place. The number of skin conductance responses in each measurement period (start, middle, end) within EM sets indicated a significant effect of time for both the first and last treatment sessions (see Table 2). In particular, the number of skin conductance responses decreased significantly from the start (the first 10 seconds) to the middle and end of the EM sets in both the first and last treatment sessions. Overall, findings indicate a reduction in the number of skin conductance responses within EM sets.

The amplitude of the skin conductance responses in each measurement period within EM sets indicated a significant effect of time for the last treatment session but a nonsignificant effect of time for the first treatment session (see Table 2). In particular, in the last treatment session, there was a significant reduction in 


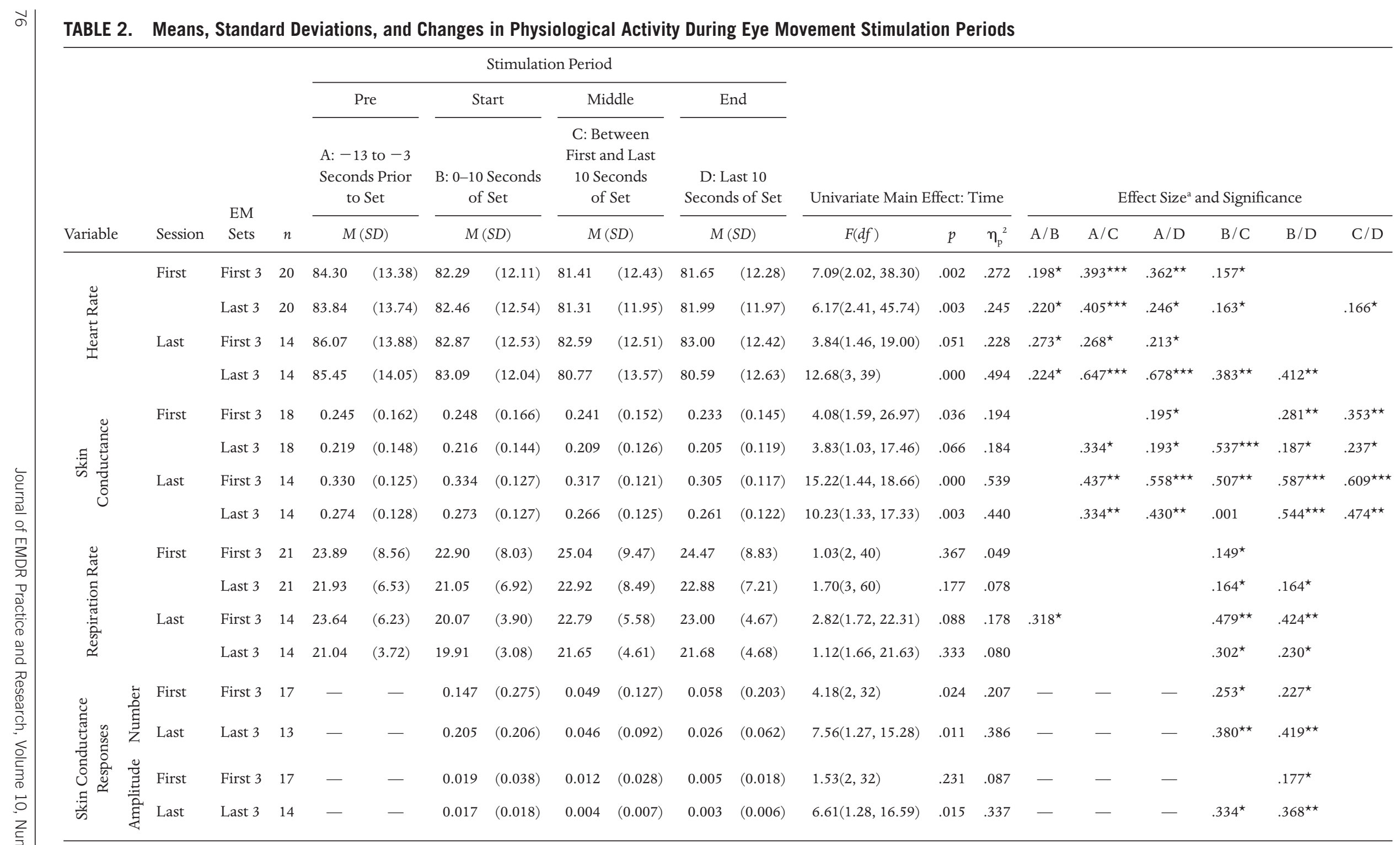

Note. $\mathrm{EM}=$ eye movement.

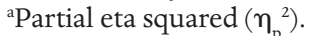

${ }^{\star} p<.05 .{ }^{\star \star} p<.01 .{ }^{\star{ }^{\star}} p<.001$. 
the size of skin conductance responses from the start compared to the middle and end of the EM sets.

\section{Discussion}

This study examined the physiological correlates of EMDR therapy when effectively used to treat adults with PTSD symptoms in Timor-Leste: a postwar/ conflict, developing nation. As previously reported, the EMDR treatment was followed by significant reductions in PTSD, depression, and anxiety symptoms (Schubert et al., 2016). Hypotheses regarding psychophysiological changes during EMDR sessions were partly supported in that trends and significant decreases in heart rate, respiration rate, and skin conductance indicated physiological dearousal within treatment sessions; physiological responses associated with the EMs included a significant decrease in heart rate as EMs began; and heart rate and skin conductance decreased within EM sets. In addition, skin conductance responses habituated in number and size within EM sets characteristic of an orienting response. However, although there was a trend for respiration rate to increase in EM sets, the hypotheses that respiration rate would increase significantly during EM sets was not supported.

\section{Psychophysiological Changes From Before to After Desensitization Sessions}

In general, physiological data in this study suggested that arousal decreased within EMDR desensitization sessions. Resting levels from before to after the first desensitization session decreased significantly in heart and respiration rate, and skin conductance levels decreased significantly in the last desensitization session. Physiological de-arousal after traumafocused therapy has previously been reported in Western settings when EMDR therapy (Elofsson et al., 2008; Sack, Lempa, et al., 2008) and therapeutic exposure (Boudewyns \& Hyer, 1990) has been used effectively to treat PTSD. Although this real-world study had no comparison control, the trends and significant de-arousal seen in Timorese participants support previous controlled findings; for example, single EMDR sessions used to treat distressing traumatic memories led to greater pre- to postreductions in heart rate and galvanic skin response compared to two comparison conditions (Wilson et al., 1996); EMDR led to significant reductions in heart rate and skin conductance responses to trauma recall com- pared to a relaxed state (Aubert-Khalfa, Roques, \& Blin, 2008); and decreases in heart rate and increases in heart rate variability indicated increased parasympathetic tone during exposure to script-driven imagery compared to a neutral task after EMDR and at 6 months follow-up for PTSD patients (Sack, Lempa, \& Lamprecht, 2007). Changes were also similar to those seen during EMDR treatment of a negative autobiographical memory in a nonclinical population (Schubert et al., 2011).

\section{Psychophysiological Changes During \\ Eye Movement Stimulation}

The physiological changes during EM stimulation in this study are consistent with previous research that have demonstrated that EM sets are associated with a pattern of de-arousal coupled with distinct physiological changes that may aid memory processing (Elofsson et al., 2008; Sack, Lempa, et al., 2008; Schubert et al., 2011). In Timorese participants, we saw a clear, significant drop in heart rate when EMs began, confirming observations in Western settings of patients with PTSD (Elofsson et al., 2008; Sack, Lempa, et al., 2008) and in a nonclinical population where EMDR was used to treat negative autobiographical memories (Schubert et al., 2011). The decrease in heart rate as EMs began has been attributed to an orienting response (Sack, Lempa, et al., 2008) specific to the EMs in EMDR because the immediate, pronounced heart rate decrease was not present when EMs were removed from the therapy procedure (Schubert et al., 2011).

Our data also indicated that heart rate, along with skin conductance, decreased within EM sets. This decrease in heart rate and skin conductance has been consistently reported in studies of physiological activity during EMDR (Elofsson et al., 2008; Sack, Lempa, et al., 2008; Schubert et al., 2011; Wilson et al., 1996). Skin conductance data in this study also mirrors previous reports (Elofsson et al., 2008; Schubert et al., 2011) in that skin conductance decreased significantly only from the middle, rather than start, of EM sets. In principle, if the prominent decrease in heart rate at the start of EM sets is driven by an orienting response then it should be coupled with an immediate startle response indexed by skin conductance responses in the first 10 seconds after EMs begin (Bradley, 2009), which then habituate. This pattern of response was seen in this study because there were significantly more and larger skin conductance responses at the beginning of EM sets than during the middle and end of sets. 
In the first physiological examination of EMDR sessions 20 years ago, it was reported that "during EMDR, the GSR [galvanic skin response] characteristically rose at the beginning of the set-as the subject focused on a disturbing memory-peaked at 5-10 seconds, then decreased thereafter" (Wilson et al., 1996, p. 226). Wilson (1996) proposed that this galvanic skin response rise and fall was distinct from an orienting response as in their study; they reported that EMs alone, without focusing on a traumatic memory, did not produce an increase in galvanic skin response followed by decrease. However, more recently, Schubert et al. (2011) found that EMDR-with-EMs produced significantly more skin conductance responses within desensitization sets than EMDR-without-EMs; the amplitude of the skin conductance responses decreased significantly within EM sets in EMDR-with-EMs compared to EMDR-without-EMs; and the number of skin conductance responses habituated across the session for EMDR-with-EMs compared to no significant change for the no-EM condition. These findings, combined with the present confirmatory data in Timorese participants, in which skin conductance responses reduced in number and size within EM sets, along with the immediate decrease in heart rate as EMs began, and the decrease in heart rate and skin conductance across EM sets, suggest that EMs in EMDR elicit an orienting response.

The role of an orienting response in EMDR is theoretically important. From an orienting response perspective, physiological changes reflect specific perceptual and motor processes that have evolved to protect the organism from threat (Bradley, 2009). Heightened skin conductance is an index of sympathetic nervous system activation, a defense response supporting behavioral arousal and preparation for action, whereas cardiac deceleration is associated with enhanced perceptual processing, an openness to intake of sensory information (Bradley, 2009). From dismantling laboratory research, the type of EMs used in EMDR have been shown to facilitate attentional orienting and with that induce cognitive and semantic flexibility (Kuiken, Bears, Miall, \& Smith, 2001) and also enhance episodic memory retrieval (Christman, Garvey, Propper, \& Phaneuf, 2003). A meta-analysis (Lee \& Cuijpers, 2013) that examined the effects of EMs in both laboratory and EMDR treatment studies confirmed that EMs in both settings significantly decreased vividness and, in turn, the distress of negative memories (Smeets, Dijs, Pervan, Engelhard, \& van den Hout, 2012). Neuroimaging research that examined changes in brain activity pre- and post-EMDR treatment (Levin, Lazrove, \& van der Kolk, 1999), along with Stickgold (2002, 2008), who first recognized the neurological and physiological pathways linking EMDR to REM functioning, states that EMDR therapy creates neurological states that facilitate memory processing. Although it cannot be simply assumed that EMs in laboratory, dismantling studies create the same neural environment as EMs in EMDR therapy during desensitization of trauma memories; nevertheless, in EMDR, the EMs, and the associated orienting responses, may be important for attending to and making sense of memories, thoughts, feelings, and bodily sensations that arise during desensitization of traumatic memories. From the treatment studies that have examined the physiological correlates of EMDR therapy (Elofsson et al., 2008; Sack, Lempa, et al., 2008; Wilson et al., 1996), it can be stated that the EMs and associated orienting responses induce a form of psychophysiological de-arousal that is incompatible with fear and avoidance, which may make the task of attending to and processing trauma and associated memories more tolerable.

REM hypotheses of EMDR propose that orienting responses, induced by the EMs in therapy, activate brainstem circuits that could initiate a REM-like state (Stickgold, 2002). Previous research has found similarities in physiological changes during the EMs in EMDR compared to the typical autonomic pattern of REM sleep (Elofsson et al., 2008). For example, during EMs in EMDR, there was a change in respiration rate manifested by increased frequency and a tendency for participants to become more hypercapnic (increased carbon dioxide) and hypoxemic (decreased levels of oxygen; Elofsson et al., 2008). This respiratory profile is consistent with REM sleep. Other researchers have found that the EMs in EMDR are associated with an increase in respiration within EM sets which may suggest similar physiological states during EMDR and REM because REM sleep has been associated with fast, shallow breathing (Elofsson et al., 2008; Sack, Lempa, et al., 2008; Schubert et al., 2011). In this study, although there was a trend in the expected direction, the increase in respiration during EMs was not significant. Although this may be caused by the small sample size, the measurement of respiration used in TimorLeste was also different from Elofsson and colleagues (2008) who measured expiratory carbon dioxide levels (via a nasal catheter) and blood pulse oximeter oxygen saturation, with respiration rate computed using recorded carbon dioxide wave data. In this study, respiration was measured using a self-inflating pressure pad transducer around the participant's chest because 
this was considered nonintrusive to the therapy process. The cost, however, was that raw respiratory data was markedly influenced by artifact, and as in previous research (Schubert et al., 2011), respiration rate was determined by manually counting and averaging respiration cycles for each measurement period. Thus, respiration data in this study relied on human judgment. Future research would benefit from replicating the respiration findings within Elofsson et al. (2008) and Sack, Lempa, et al. (2008), whose measures did not introduce subjectivity.

Limitations were that this study was a real-life, naturalistic intervention without a separate control condition. One cannot, therefore, rule out that the physiological changes were caused by something other than the treatment. Psychophysiological data in this preliminary study need to be interpreted with caution because of the small sample size and variation in the number of participants across measurement periods. Further research to confirm these findings is warranted, using a larger sample size with active controls and a wider range of physiological variables. For example, future protocols should also incorporate finger temperature (or a more dynamic measure of vascular tone) to explore whether REM-like mechanisms are involved and, if so, to differentiate between the mechanisms of EMs being orienting response and/or REM-related. To examine REM hypotheses of EMDR further, it would be interesting to determine whether the startle responses associated with the EMs in EMDR occur in concert with brainstem-initiated ponto-geniculo-occipital (PGO) waves because REM sleep is the only state known to generate PGO waves, or rather, PGO waves are theorized to signal the beginning of REM-sleep cycle (Hobson, Stickgold, \& Pace-Schott, 1998; Stickgold, 2002). In addition to orienting response theory and REM effects, working memory (Maxfield, Melnyk, \& Hayman, 2008) and neurological reconsolidation (Levin et al., 1999), along with processes such as reciprocal inhibition (Wolpe, 1991) and psychological distancing (Lee \& Drummond, 2008) may contribute to EMDR and require consideration in future research to attain a comprehensive understanding of how EMDR works.

\section{Conclusion}

To our knowledge, this is the first research that has examined physiological correlates of EMDR therapy when used to treat trauma symptoms in a realworld setting, postwar/ conflict, developing country. The psychophysiological changes seen in Timorese participants resembled those seen in Western treatment settings and may aid in the refinement of models that attempt to explain the underlying basis of EMDR therapy. Because trauma is a global issue, the examination of effective trauma therapy ought to be global and cross-cultural, and evidence should be sought in all contexts and populations as to how the body and brain mechanisms lead to trauma recovery.

\section{References}

Aubert-Khalfa, S., Roques, J., \& Blin, O. (2008). Evidence of a decrease in heart rate and skin conductance responses in PTSD patients after a single EMDR session. Journal of EMDR Practice and Research, 2, 51-56. http:/ / dx.doi .org/10.1891/1933-3196.2.1.51

Australian Centre for Posttraumatic Mental Health. (2013). Australian guidelines for the treatment of acute stress disorder and posttraumatic stress disorder. Melbourne, Australia: Author.

Boudewyns, P. A., \& Hyer, L. (1990). Physiological response to combat memories and preliminary treatment outcome in Vietnam veteran PTSD patients treated with direct therapeutic exposure. Behavior Therapy, 21, 63-87. http: / / dx.doi.org/10.1016/S0005-7894(05)80189-3

Bradley, M. M. (2009). Natural selective attention: Orienting and emotion. Psychophysiology, 46, 1-11. http://dx.doi .org/10.1111/j.1469-8986.2008.00702.x

Cahill, S. P., Carrigan, M. H., \& Frueh, B. C. (1999). Does EMDR work? And if so, why?: A critical review of controlled outcome and dismantling research. Journal of Anxiety Disorders, 13, 5-33. http:/ / dx.doi.org/10.1016/ S0887-6185(98)00039-5

Christman, S. D., Garvey, K. J., Propper, R. E., \& Phaneuf, K. A. (2003). Bilateral eye movements enhance the retrieval of episodic memories. Neuropsychology, 17, 221-229. http: / / dx.doi.org/10.1037/0894-4105.17.2.221

Davidson, P. R., \& Parker, K. C. H. (2001). Eye movement desensitization and reprocessing (EMDR): A meta-analysis. Journal of Consulting and Clinical Psychology, 69, 305-316. http: / / dx.doi.org/10.1037 / 0022-006X.69.2.305

Elofsson, U. O. E., von Schéele, B., Theorell, T. R., \& Söndergaard, H. P. (2008). Physiological correlates of eye movement desensitization and reprocessing. Journal of Anxiety Disorders, 22, 622-634. http://dx.doi .org/10.1016/j.janxdis.2007.05.012

Hobson, J. A., Stickgold, R., \& Pace-Schott, E. F. (1998). The neuropsychology of REM sleep dreaming. Neuroreport, 9, R1-R14.

Kuiken, D., Bears, M., Miall, D., \& Smith, L. (2001). Eye movement desensitization reprocessing facilitates attentional orienting. Imagination, Cognition and Personality, 21, 3-20. http://dx.doi.org/10.2190/ L8JX-PGLC-B72R-KD7X 
Lee, C. W., \& Cuijpers, P. (2013). A meta-analysis of the contribution of eye movements in processing emotional memories. Journal of Behavior Therapy and Experimental Psychiatry, 44, 231-239. http://dx.doi.org/10.1016/ j.jbtep.2012.11.001

Lee, C. W., \& Drummond, P. D. (2008). Effects of eye movement versus therapist instructions on the processing of distressing memories. Journal of Anxiety Disorders, 22, 801-808. http://dx.doi.org/10.1016/j.janxdis .2007.08.007

Levin, P., Lazrove, S., \& van der Kolk, B. (1999). What psychological testing and neuroimaging tell us about the treatment of posttraumatic stress disorder by eye movement desensitization and reprocessing. Journal of Anxiety Disorders, 13, 159-172. http://dx.doi.org/10.1016/ S0887-6185(98)00045-0

Maxfield, L., Melnyk, W. T., \& Hayman, C. A. G. (2008). A working memory explanation for the effects of eye movements in EMDR. Journal of EMDR Practice and Research, 2, 247-261. http: / / dx.doi.org/10.1891/ 1933-3196.2.4.247

Modvig, J., Pagaduan-Lopez, J., Rodenburg, J., Salud, C. M. D., Cabigon, R. V., \& Panelo, C. I. A. (2000). Torture and trauma in post-conflict East Timor. Lancet, 356, 1763. http: / / dx.doi.org/ 10.1016/S0140-6736(00)03218-9

Mollica, R. F., Caspi-Yavin, Y., Bollini, P., Troung, T., Tor, S., \& Lavelle, J. (1992). The Harvard Trauma Questionnaire. Validating a cross-cultural instrument for measuring torture, trauma, and posttraumatic stress disorder in Indochinese refugees. The Journal of Nervous and Mental Disease, 180, 111-116.

Oren, E., \& Solomon, R. (2012). EMDR therapy: An overview of its development and mechanisms of action. European Review of Applied Psychology, 62, 197-203. http:/ / dx.doi.org/10.1016/j.erap.2012.08.005

Pole, N. (2007). The psychophysiology of posttraumatic stress disorder: A meta-analysis. Psychological Bulletin, 133, 725-746.http:/ / dx.doi.org/10.1037/0033-2909.133.5.725

Raboni, M. R., Tufik, S., \& Suchecki, D. (2006). Treatment of PTSD by eye movement desensitization reprocessing (EMDR) improves sleep quality, quality of life, and perception of stress. Annals of the New York Academy of Sciences, 1071, 508-513. http://dx.doi.org/10.1196/annals .1364 .054

Sack, M., Hofmann, A., Wizelman, L., \& Lempa, W. (2008). Psychophysiological changes during EMDR and treatment outcome. Journal of EMDR Practice and Research, 2, 239-246. http: / / dx.doi.org/10.1891/ 1933-3196.2.4.239

Sack, M., Lempa, W., \& Lamprecht, F. (2007). Assessment of psychophysiological stress reactions during a traumatic reminder in patients treated with EMDR. Journal of EMDR Practice and Research, 1, 15-23. http://dx.doi .org/10.1891/1933-3196.1.1.15

Sack, M., Lempa, W., Steinmetz, A., Lamprecht, F., \& Hofmann, A. (2008). Alterations in autonomic tone during trauma exposure using eye movement desensitization and reprocessing (EMDR)—Results of a preliminary investigation. Journal of Anxiety Disorders, 22, 1264-1271. http:/ / dx.doi.org/10.1016/j.janxdis.2008.01.007

Schnyder, U., Ehlers, A., Elbert, T., Foa, E. B., Gersons, B. P., Resick, P. A., . . Cloitre, M. (2015). Psychotherapies for PTSD: What do they have in common? European Journal of Psychotraumatology, 6, 28186. http://dx.doi .org/10.3402/ ejpt.v6.28186

Schubert, S. J., Lee, C. W., De Araujo, G., Butler, S. R., Taylor, G., \& Drummond, P. (2016). The effectiveness of eye movement desensitization and reprocessing (EMDR) to treat symptoms following trauma in Timor Leste. Journal of Traumatic Stress. http://dx.doi.org/10.1002/ jts. 22084

Schubert, S. J., Lee, C. W., \& Drummond, P. (2011). The efficacy and psychophysiological correlates of dualattention tasks in eye movement desensitization and reprocessing (EMDR). Journal of Anxiety Disorders, 25, 1-11. http:/ / dx.doi.org/10.1016/j.janxdis.2010.06.024

Shapiro, F. (2001). Eye movement desensitization and reprocessing: Basic principles, protocols and procedures (2nd ed.). New York, NY: Guilford Press.

Smeets, M. A. M., Dijs, M. W., Pervan, I., Engelhard, I. M., \& van den Hout, M. A. (2012). Time-course of eye movement-related decrease in vividness and emotionality of unpleasant autobiographical memories, Memory, 20, 346-357. http://dx.doi.org/10.1080/09658211.2012 .665462

Sokolov, E. N., \& Cacioppo, J. T. (1997). Orienting and defense reflexes: Vector coding and the cardiac response. In P. J. Lang, R. F. Simons, \& M. Balaban (Eds.), Attention and orienting: Sensory and motivational processes (pp. 1-22). Mahwah, NJ: Lawrence Erlbaum Associates.

Söndergaard, H. P., \& Elofsson, U. (2008). Psychophysiological studies of EMDR. Journal of EMDR Practice and Research, 2, 282-288. http://dx.doi.org/10.1891/ 1933-3196.2.4.282

Stickgold, R. (2002). EMDR: A putative neurobiological mechanism of action. Journal of Clinical Psychology, 58, 61-75. http: / / dx.doi.org/10.1002/jclp.1129

Stickgold, R. (2008). Sleep-dependent memory processing andEMDRaction.Journal of EMDR Practice and Research, 2 , 289-299. http: / / dx.doi.org/10.1891/1933-3196.2.4.289

Wilson, D., Silver, S. M., Covi, W., \& Foster, S. (1996). Eye movement desensitization and reprocessing: Effectiveness and autonomic correlates. Journal of Behavior Therapy and Experimental Psychiatry, 27, 219-229. http:/ / dx.doi.org/10.1016/S0005-7916(96)00026-2

Wolpe, J. (1991). The practice of behavior therapy (4th ed.). New York, NY: Pergamon Press.

World Health Organization. (2013). Guidelines for the management of conditions specifically related to stress. Geneva, Switzerland: Author. Retrieved from http:/ / www.who.int/mental_health/emergencies/stress_ guidelines/en/

Wright, R. D., \& Ward, L. M. (2008). Orienting of attention. New York, NY: Oxford University Press. 
Acknowledgments. The trial was registered with the Australian New Zealand Clinical Trials Registry (ANZCTR): 12611000239965. Guilhermina de Aroujo, Susan Butler, Dilyana Datten, and Graham Taylor are gratefully acknowledged for their valuable help during planning and data collection, and laboratory technician, Man Trac. The Anxiety Disorders Foundation of Western Australia and the EMDR Research Foundation are acknowledged for their appreciated support of this project. Thank you to the staff of the Timorese nongovernmental organizations,
Psychosocial Recovery and Development in East Timor, Australian People for Health, Education and Development Abroad, and Ahisaun Disability Foundation. Insightful comments by two anonymous reviewers were also greatly appreciated.

Correspondence regarding this article should be directed to Sarah J. Schubert, 90 Selway Road, Brentwood, WA, Australia, 6153. E-mail: s.schubert@murdoch.edu.au 